to eight and there are two other buttons, nine and zero, so that a man can supply numerical data such as his age and check number. There is also a button he can use to cancel a wrong answer and a button which switches on a microphone and tape-records any verbal comments that may be necessary.

The sets of pictures and words are recorded on film and projected on to the screen by the optical device of a standard teaching machine, but the system for presenting the questions needs to be much faster and more flexible than that used in normal machines of this type. The circuitry and the question sequence are such that the answer button a man presses also determines which question he is asked next. He may simply be asked the next question on the film, but there is a range of other possibilities, and it can sometimes happen that the operation of each of the eight principal answer buttons introduces a different question. Thus though the machine may be able to ask more than a thousand questions covering different circumstances and types of accident, it will by-pass irrelevant questions and ask only the limited number of questions that apply to the accident concerned.

Each answer is recorded in a form that can be automatically transferred to paper tape. When necessary, the operator transferring this information can make any modifications that result from the verbal comments of the injured man. The resulting paper tapes are in a form immediately ready for computer analysis.

\title{
NEW APPLIANCES
}

\section{Heat-retaining Mattress for Temperature Control in Surgery}

Dr. A. F. WINDER, lecturer in pharmacology, and Dr. R. J. VALE, consultant anaesthetist, Guy's Hospital, London S.E.1, write: Errors of heat balance in anaesthetized surgical patients result in undesirable circulatory and metabolic disturbances (Lunn, 1969). A mattress has been developed to improve thermal control in major surgery where prolonged exposure and extracorporeal circulatory techniques in air-conditioned environments lead to severe heat loss.

The mattress illustrated is made of 0.020 in. $(0.5-\mathrm{mm}$.) polyvinylchloride (P.V.C.) and has five sections to conform generally to the supine adult, the head not being supported. The undersurface has two thermometer pockets in $0 \cdot 007$-in. $(0 \cdot 18-\mathrm{mm}$.) P.V.C. and five filling points, and the mattress can be secured to an operating-table or hospital bed by 18 -in. $(46-\mathrm{cm}$.) flaps at each end ; other flaps from the lower midsection secure the legs. Overall dimensions excluding flaps are 66 by $21 \frac{1}{2}$ in. (168 by $55 \mathrm{~cm}$.). The mattress can be supplied and transported containing a dry powder, and hot water is added on arrival to produce a viscous gel, giving a final depth of 2 in. $(5 \mathrm{~cm}$.). The gel incorporates a preservative and is stable after about 48 hours. Small holes made-for example, with towel clips-are then to some extent self-sealing. The mattress is radiotranslucent and compatible with $x$-ray techniques but is not constructed of antistatic materials, and thus is not approved for use in conjunction with explosive anaesthetic agents.

Operation.-The mattress is prepared for use by preheating overnight to the required temperature with a thermostatically controlled electric blanket covered with a heat reflecting blanket (space blanket). This temperature is generally $33-35^{\circ}$ C.: Benzinger et al. (1963) showed that body heat production and oxygen consumption increase when the average skin temperature falls below $33^{\circ} \mathrm{C}$. The temperature is checked by inserting thermometers into the underpockets or with upper and lower surface thermistors. Temperature-sensitive coloured discs can also be attached to the surfaces. The correct temperature must be carefully achieved, but once prepared the mattress requires no furthe attention or accessory equipment while in use and will not produce burns. In the present trials the patient is transferred to the mattress on leaving the ward and remains on it during operation and for two or three days postoperatively.

Performance.-The useful thermal and

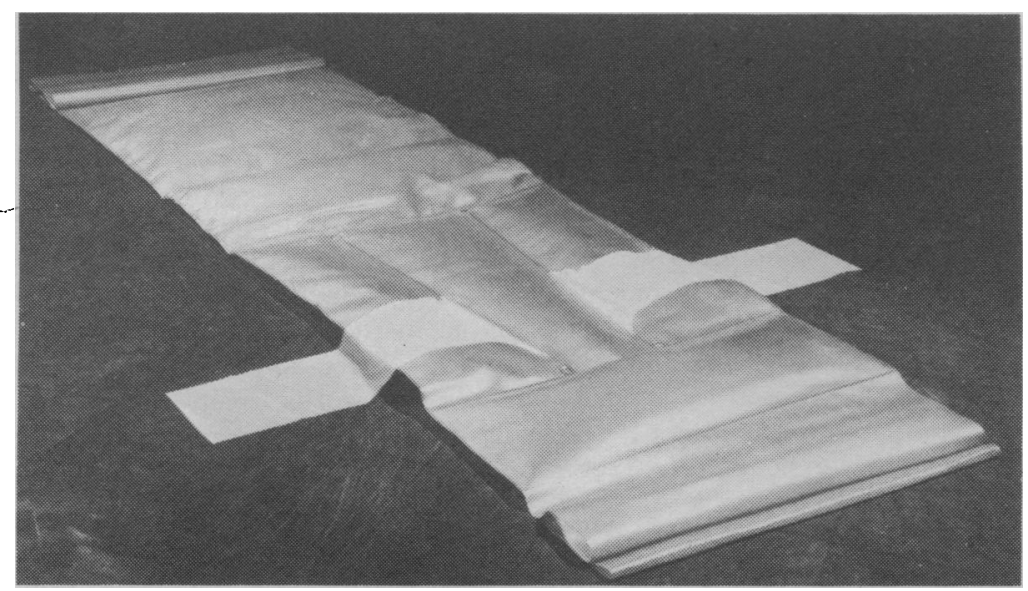

mechanical properties of the mattress are inherent in the highly viscous aqueous gels it contains, which combine high specific heat, and thus heat content, with low thermal conductivity and rate of heat transfer. Our experiments with a range of methyl cellulose gels have shown that specific heats in excess of 0.97 are compatible with viscosity coefficients in excess of 10,000 poise at $20^{\circ} \mathrm{C}$ (water 0.010 poise). This high viscosity allows distributed support for the body in comfort, with good surface contact for heat transfer, and cooling curves determined with the mattress show reduced rates of heat loss -that is, transfer-in comparison with the identical water-filled device. Heat transfer is bidirectional and thus the mattress functions as a thermal buffer, allowing changes in the patient's temperature to be compensated by transfer to or from the mattress. This view is supported by Vale and Lunn (1969), who describe stabilization of skin and nasopharyngeal temperatures in a group of patients undergoing open heart surgery on the mattress.

Application.-The mattress was developed to improve heat balance in major surgery in adults and children, but other interesting applications have recently become evident. The resistance to change in temperature from the preset value suggests that it may assist the maintenance of hypothermia. The mechanical properties indicate that the mattress may contribute to the prevention of bedsores, and a trial is now in progress.

The above form of mattress is the subject of U.K. Patent application No. 51624/68, and is now available from Perfusion Associates Ltd., 18 Macaulay Road, London S.W.4

REFERENCES

Benzinger, T. H., Kitzinger, C., and Pratt, A. W. 1963). In Temperature-Its Measurement and Control in Science and Industry, vol. 3, edited Reinhold. Lunn, H. F. (1969). Guy's Hospital Reports, 118, 117 